\title{
Increasing rates of suicide across cultures
}

\section{David Skuse}

Behavioural and Brain Sciences Unit, Institute of Child Health, London WC1 1EH, UK, email d.skuse@ich.ucl.ac.uk

uicide, especially among young people, appears to be increasing in prevalence in diverse countries and cultures. The reasons for this worrying trend remain obscure. Here we present commentaries on suicidal trends in three countries.

First, Masahito Fushimi and colleagues from Japan draw our attention to the dramatic increase in the number of suicides, especially in Akita Prefecture, where the rate is double the national average. It is extraordinary to read that not only was the highest suicide rate among those of middle age, but the cause of death was usually by hanging, a mode of suicide that is rather unusual outside certain penal institutions in the UK. Economic worries are thought to be an important aetiological influence; they have been exacerbated by the 15 years in which Japan has experienced debt and deflation-ridden economic stagnation. There is also a worrying trend for younger people to engage in suicide pacts made via the internet, a vogue that is now worrying countries in the West as well.

Brazil is a diverse and vibrant country with very different cultures and huge discrepancies in wealth, both within and between regions. The suicide rate is apparently astonishingly low by international comparison, although the country's religious culture may mean that the reported rate is an underestimate. Even so, there has been an increasing risk of suicide over the last two decades, especially among young people. Carolina de Mello-Santos and colleagues discuss this trend, which, in terms of preferred method, is linked especially to the wide availability of firearms (although poisoning and hanging are also common). Increasingly, young single males with low educational attainments and poor economic prospects are the victims.

Finally, $\operatorname{Dr}$ N. K. Ndosi provides a fascinating account of suicides in Africa, from the perspective of Tanzania. In Africa, we are told, there are very strong societal prohibitions against suicide, which brings opprobrium on the family of the victim. Despite these attitudes, there is a trend to increasing suicidal behaviour in sub-Saharan Africa, especially among the young, which could be related to increasing urbanisation and the breaking down of traditional cultural structures which militated against the behaviour in former times. We find not only aetiological factors that are culturally relatively specific, such as the oppression of women in patriarchal societies, but also the creeping in of those that were formerly concerns within deprived inner cities in the West, such as heroin addiction. The role to be played by psychiatrists in the primary and secondary prevention of suicide in the diverse cultures discussed in these articles is discussed by all contributors.

\section{Suicide in Akita Prefecture, Japan}

\section{Masahito Fushimi, ${ }^{1,2}$ Junya Sugawara ${ }^{3}$ and Tetsuo Shimizu ${ }^{3}$}

${ }^{1}$ Akita Prefectural Mental Health and Welfare Center, Daisen City, Akita 019-2413, Japan,

email fushimi@pref.akita.lg.jp

${ }^{2}$ Akita Prefectural Rehabilitation and Psychiatric Center, Akita, Japan

${ }^{3}$ Department of Psychiatry, Akita University School of Medicine, Akita, Japan

$\mathrm{n}$ recent years, the number of suicides in Japan has increased dramatically, particularly among middleaged men. According to the Brief Report on Suicides in 200 I by the National Police Agency of Japan (NPA, 2002), the number of suicides in Japan was 31042 , and the national suicide rate was 24.4/100000. Akita is an agricultural prefecture with a population of approximately I 200000 . According to the Akita Prefectural Police (APP), the number of suicides in Akita Prefecture was 457 (299 males, I 58 females) in 2001. Akita Prefecture currently has the highest rate of suicide in Japan. The identification of strategies for suicide prevention is therefore imperative.
Psychological autopsy is useful for obtaining background information regarding suicide victims (Conwell et al, 1996; Cheng et al, 2000). However, obtaining consent for such studies from the families of the deceased is difficult in Japan. As members of the Akita Prefectural Medical Association (APMA) are thought to have many opportunities to obtain background information on suicides, an investigation of suicides in which members of the APMA are consulted might prove advantageous for accumulating representative data from a large number of cases. The present report describes the results of a questionnaire about suicide that was distributed to members of the APMA
It is extraordinary

to read that not only was the

highest suicide rate among those of middle age, but the cause of death was usually by hanging, a mode of suicide that is rather unusual outside certain penal institutions in the UK. 
in order to determine the factors underlying suicide in Akita Prefecture.

\section{The survey}

Members of the APMA who had attended a suicide case were asked to complete a questionnaire about it. The APMA conducted this investigation from I July 2001 to 30 June 2002. The total number of suicides during this period was 138 ( 102 males, 36 females). A major peak in the number of suicides was observed among individuals in their 50s and 60s, particularly for males. Conversely, female cases increased in number in elderly age-groups compared with male cases. The most common method of suicide was hanging, which was observed in 105 cases (78 males, 27 females). Most suicides (94 cases) were performed at home. The number of suicides according to time of day tended to increase from midnight to early morning and from daytime to evening, which would be when the rest of the family was asleep or absent. Regarding physical disorders, the majority involved chronic diseases (e.g. hypertension). Depressive disorder was the most common psychiatric disorder. Furthermore, character traits which are thought to be strongly associated with depression were common.

The most frequently observed complaints were economic problems. In addition, different age-groups tended to present with different complaints. Specifically, for individuals under 40 years of age, most complaints involved private problems other than health-related ones. For those aged 40-59 years, most complaints involved economic problems. For older adults (those aged over 60 years), most complaints involved health-related problems.

For individuals
under 40 years
of age, most
complaints
involved private
problems other
than health-related
ones. For those
aged $40-59$ years,
most complaints
involved economic
problems. For
older adults
(those aged
over 60 years),
most complaints
involved health-
related problems.

\section{Discussion}

In 1998, the number of suicides in Japan increased sharply and the annual number of suicides exceeded 30000, making suicide a significant national problem. Particularly notable was an increase in suicides among middle-aged males.

Recently, in addition to suicide among middle-aged males, suicide pacts among young people who meet via the internet ('internet group suicide' cases) have been reported frequently in Japan, though this was not part of the survey. Typically, a suicidal individual recruits others on a message board. Subsequently, in a sealed car, they take sleeping pills and light charcoal stoves (rentan). Death results from carbon monoxide poisoning. In some cases, group members send emails to inform their family or friends just before the final act, or even after, with the message relayed from an automatic mailing system. The common use of charcoal stoves suggests that groups are imitating earlier cases reported by the media.

Statistics from the NPA indicate that when all suicide victims who left a suicide note are classified according to motive, 'health-related problems' represent the leading cause of suicide, followed by 'economic and life-related problems'. Since 1998, the number of cases falling into the latter category has increased substantially, to the extent that this motive is likely to surpass 'health-related problems' as the leading cause of suicide. The NPA reported that, among all suicides in 1998, 6058 were committed because of economic difficulties, which represents an increase of $70.4 \%$ from 1997. Furthermore, the 1999 NPA report showed that for 6758 individuals $(20.4 \%$ of suicides) suicide was a result of economic difficulties, which was up $11.6 \%$ from 1998. In addition, the 2000 NPA report showed that concerns over economic difficulties accounted for 6838 suicides (21.4\%). These findings clarify a potential relationship between suicide and economic strife (e.g. financial hardships, low income, or unemployment, as a result of the deep and prolonged recession of the Japanese economy). Indeed, the popular press has focused on the correlation between the current economic situation in Japan and suicide rates. According to the APP, the number of suicides in Akita Prefecture that are a result of economic and life-related problems has increased markedly. In 2002, this motive became the leading cause of suicide by surpassing health-related problems (the leading cause until 200I). Similarly, in the present study, economic problems were shown to be most common, followed by health-related problems.

Based on the results of the present study, suicides can be categorised into two groups: one considered to be relatively amenable to medical intervention; and the other considered to be relatively resistant to it. Included in the former group are suicides caused by several psychiatric disorders (depression in particular), while the latter group includes suicides influenced by social situations such as economic problems (e.g. Japan's prolonged recession). As noted in previous studies, early detection and appropriate treatment for depression are obviously crucial for suicide prevention (Rutz et al, 1992). However, the majority of middle-aged male suicides are thought to be performed in order to settle economic problems, which reflects current social conditions. In such cases, medical approaches alone may be insufficient for suicide prevention.

The present results do not exclude the possibility that economic problems are a major factor in the recent increase in the number of suicides. However, strategies for managing depression are also considered important.

\section{References}

Cheng, A. T., Chen, T. H., Chen, C. C., et al (2000) Psychosocia and psychiatric risk factors for suicide. Case-control psychological autopsy study. British Journal of Psychiatry, 177, 360-365.

Conwell, Y., Duberstein, P. R., Cox, C., et al (1996) Relationships of age and axis I diagnoses in victims of completed suicide: a psychological autopsy study. American Journal of Psychiatry, I53, 1001-1008.

NPA (1999) Brief Report on Suicides in 1998. Tokyo: NPA (in Japanese). 
NPA (2000) Brief Report on Suicides in 1999. Tokyo: NPA (in Japanese).

NPA (200I) Brief Report on Suicides in 2000. Tokyo: NPA (in Japanese).

NPA (2002) Brief Report on Suicides in 2001. Tokyo: NPA (in Japanese).

Rutz, W., Von Knorring, L. \& Walinder J. (1992) Long-term effects of an educational program for general practitioners given by the Swedish Committee for the Prevention and Treatment of Depression. Acta Psychiatrica Scandinavica, 85, 83-88.

\section{Further reading}

Fushimi, M., Sugawara, J. \& Shimizu, T. (2005) Suicide patterns and characteristics in Akita, Japan. Psychiatry and Clinical Neurosciences, 59, 296-302.

\section{Suicide trends and characteristics in Brazil}

\section{Carolina de Mello-Santos, ${ }^{1}$ José Manoel Bertolote ${ }^{2}$ and Yuan-Pang Wang ${ }^{1,3}$}

'Psychiatry Institute of the Universidade de São Paulo School of Medicine, São Paulo, Brazil, email caro_mello@hotmail.com

${ }^{2}$ World Health Organization Team on Management of Mental and Brain Disorders

${ }^{3}$ Santo Amaro School of Medicine, UNISA, São Paulo, Brazil

razil is the largest and most populous country in South America (in 2002 the population was approximately 175 million). Although life expectancy in Brazil has increased, suicide and other forms of injury-related mortality, such as homicide and accident, have increased as a proportion of overall mortality (Oswaldo Cruz Foundation, 1984; Brazil Ministry of Health, 200I). The suicide rate in Brazil (3.0-4.0 per 100000 inhabitants) is not considered high in global terms (World Health Organization, 1999). Nevertheless, it has followed the world tendency towards growth: during 1980-2000, the suicide rate in Brazil increased by $21 \%$. Elderly people present the highest suicide rates in absolute numbers, but the alarming finding in the Brazilian data is that the youth population is increasingly dying by suicide (Mello-Santos et al, 2005). This statistic partially confirms a forecast by Diekstra \& Guilbinat (1993) that the number of deaths by suicide would dramatically increase over the next decades, mainly in developing countries, including Latin America. In these regions, socio-economic factors (such as an increase in divorce and unemployment and a decrease in religiosity) increase the risk of self-harm. We discuss the reasons for the low suicide rate in Brazil and highlight the socio-economic factors affecting its increase among the youth population in particular.

\section{Low suicide rate in Brazil: fact or artefact?}

It is important to determine whether there was an actual increase in suicide rates in Brazil or whether it represents only a methodological artefact arising from the improvement in data collection at national level. The tendency for not all deaths to be notified in Brazil is common to other Latin American countries as well; the problem is compounded by the authorities' disregard (Grossi \& Vassan, 2002). The Brazilian Mortality Information System, operated by the Ministry of Health, may be capable of tracing only about $80 \%$ of total deaths in Brazil, since $20 \%$ of deaths are not registered (Mello-Jorge et al, 1997).

Some peculiarities of the Brazilian health system may have affected the national statistics. One methodological problem of registering death is the difficulty of distinguishing suicide from other violent deaths, such as homicide and accident. Frequently, coroners do not record the basic cause of death on the death certificate, but instead explain only the nature of the lesion. In Brazil, suicide cases are registered according to the classifications in the 'external causes' section of ICD-10 (World Health Organization, 1992), which classifies lethal events not arising from biological diseases as: those resulting from violence, those resulting from fatal accidents, and suicide. This procedure hampers the gathering of conclusive data on the nature of the death, such as the non-accidental cases (e.g. exogenous intoxication in the case of alcohol-related car accidents). Brazilian authorities are more concerned with the registering of accidents and homicides than with the accuracy of suicide statistics, as homicide is the largest single cause of death in Brazil. During 1980-2002, the homicide rate in Brazil more than doubled, from II.4 per 100000 population to 28.4 (Gawryszewski \& Mercy, 2004).

Relatives and even the authorities often conceal the suicide for fear of judicial disputes or retaliation. There is a social prejudice against suicide and this results in non-disclosure (Mello-Jorge, 1988; Souza, 1991).

On the other hand, obtaining access to the means of suicide is easy in Brazil. The sale of psychotropics on the black market, the non-regulated and illegal carrying of guns, the ambiguous policy in relation to alcoholism, and the sale of caustic soda and rat poison in supermarkets are some examples. These
Elderly people

present the

highest suicide

rates in absolute

numbers, but the alarming finding in

the Brazilian data

is that the youth

population is

increasingly dying

by suicide.

Brazilian

authorities

are more

concerned with

the registering

of accidents

and homicides

than with the

accuracy of

suicide statistics,

as homicide is

the largest single

cause of death in

Brazil. 